\section{Regionalização e desenvolvimento humano: uma proposta de tipologia de Regiões de Saúde no Brasil}

\author{
Regionalization and human development: \\ a typology of health regions in Brazil
}

\author{
Regionalización y desarrollo humano: \\ una clasificación para las regiones de \\ salud brasileñas
}

Cristina Maria Rabelais Duarte 1,2

Marcel de Moraes Pedroso 1

Jaime Gregório Bellido 1

Rodrigo da Silva Moreira 1

Francisco Viacava ${ }^{1}$

\footnotetext{
1 Instituto de Comunicação e Informação Científica e Tecnológica em Saúde, Fundação Oswaldo Cruz, Rio de Janeiro, Brasil.

2 Faculdade de Medicina de Petrópolis, Petrópolis, Brasil.

Correspondência

C. M. R. Duarte

Laboratório de Informação

em Saúde, Instituto de

Comunicação e Informação

Científica e Tecnológica em

Saúde, Fundação

Oswaldo Cruz.

Av. Brasil 4365, Pavilhão Haity

Moussatché, Rio de Janeiro, $R J$

21040-360, Brasil.

crisrabelais@gmail.com
}

\begin{abstract}
This article aimed to present a proposal for characterizing health regions in Brazil based on human development, contributing to the identification of comparable geographic areas for observation, analysis, and monitoring of performance in regionalized health systems. The dimensions of the Municipal Human Development Index were calculated for the health regions by aggregating data from municipalities, weighted by population size. The grouping of health regions in 5 groups, based on combinations of life expectancy, income, and schooling, was determined by the K-Means method. Approximately half of Brazil's health regions were classified as type 1 and the other half as types 3 to 5. The typology provides a clustering model for homogeneous health regions, consistent with the theoretical assumptions of PROADESS. The choice of wellestablished indicators and aggregation methods tends to facilitate their comprehension and use by the actors involved in the administration of the Brazilian Unified National Health System (SUS).
\end{abstract}

Regional Health Planning; Health Evaluation; Human Development

\section{Resumo}

O objetivo do trabalho foi apresentar uma proposta de diferenciação das Regiões de Saúde no Brasil, baseada no desenvolvimento humano, contribuindo para a identificação de espaços geográficos comparáveis para observação, análise e acompanhamento do desempenho dos sistemas regionalizados de saúde. Os valores das dimensões do Índice de Desenvolvimento Humano Municipal foram calculados para as Regiões de Saúde pela agregação dos dados dos municípios, ponderados pelo seu tamanho populacional. $O$ agrupamento das Regiões de Saúde em 5 grupos, segundo combinações de longevidade, riqueza e escolaridade, foi determinado pelo método KMédias. Metade das Regiões de Saúde brasileiras foi classificada em grupos do tipo 1 e 2 e a outra metade em grupos dos tipos 3 a 5 . A tipologia apresentada oferece um modelo de agrupamento de Regiões de Saúde homogêneas, coerente com os pressupostos teóricos do PROADESS. A opção por indicadores e métodos de agregação bem estabelecidos tende a favorecer a sua compreensão e utilização pelos atores ligados à gestão do Sistema Único de Saúde (SUS).

Regionalização; Avaliação em Saúde; Desenvolvimento Humano 
Sistemas de atenção à saúde devem se constituir em respostas às necessidades de saúde dos cidadãos, operando em coerência com a situação de saúde da população usuária. No Brasil, a estruturação em uma rede regionalizada e hierarquizada de atenção à saúde é a principal característica que norteia a organização do Sistema Único de Saúde (SUS), desde a promulgação da Constituição Federal 1 e das Leis Orgânicas da Saúde (Lei no $8.080^{2}$, complementada pela Lei no $8.142^{3}$ ).

Apesar do avanço que a constituição do SUS representou em relação a períodos anteriores no país, a organização de uma rede regionalizada e hierarquizada continua sendo um dos seus principais desafios, especialmente frente à persistência de desigualdades no perfil sanitário e à desassociação frequentemente observada entre as necessidades de saúde e o acesso à rede de serviços necessária ao atendimento integral.

A situação de saúde brasileira é marcada, atualmente, pela predominância de doenças e agravos não transmissíveis (DANT), por uma carga de morbimortalidade importante relacionada às causas externas e pela ocorrência ainda não superada de doenças infecciosas e negligenciadas. Como lembra Mendes 4 (p. 18), essa situação não pode ser adequadamente respondida por um sistema de atenção à saúde "fragmentado, reativo, episódico e voltado prioritariamente para o enfrentamento das condições agudas e das agudizações das condições crônicas”. É necessário restabelecer a coerência entre a situação de saúde e o SUS, o que envolve a implantação das redes de atenção à saúde "organizadas em sistemas integrados que permitam responder, com efetividade, eficiência, segurança, qualidade e equidade, às condições de saúde da população brasileira".

Na última década e mais agudamente nos últimos anos, o foco sobre a regionalização tem-se intensificado tanto no que se refere à produção acadêmica, com o aprofundamento dos estudos sobre as peculiaridades e perspectivas da regionalização brasileira, como em relação às políticas públicas, especialmente após a instituição do Decreto no 7.508 5, regulamentador da Lei no 8.080, em junho de 2011.

No cenário atual, a criação de Regiões de Saúde está subordinada à pactuação entre o governo estadual e os governos dos municípios que compõem cada região e a acordos horizontais entre os secretários municipais de saúde. Depende, em grande medida do espírito de cooperação dos entes que compõem o "federalismo sanitário brasileiro" ${ }^{6}$. Sob a égide do Decreto no 7.508, o processo de regionalização assume um maior grau de complexidade e se associa a uma percepção mais ampla e integrada de transformação socioeconômica e de redução das desi- gualdades regionais, sendo amparada pela expansão do investimento público federal, estadual e municipal 7 .

Na busca pela concretização dos ditames legais, ganha especial relevo a utilização de modelos de monitoramento e avaliação do desempenho do sistema de saúde regionalizado, que possam municiar o planejamento em todos os níveis de gestão do SUS.

Apesar de, nas últimas décadas, uma profusão de definições e modelos de avaliação em saúde tenha sido proposta ${ }^{8}$, no cenário mundial, o lançamento do World Health Report ${ }^{9}$ pela Organização Mundial da Saúde (OMS) em 2000 representa um marco. $\mathrm{O}$ modelo proposto, baseado na construção de um indicador composto pelo qual foi elaborado um ranking de 191 países, foi duramente criticado pela comunidade científica internacional. Entretanto, sua principal inovação foi tratar da questão da avaliação do desempenho dos sistemas de saúde no mundo, incluindo uma perspectiva comparativa. O impacto positivo provocado pela proposição de um quadro conceitual para avaliar o desempenho de sistemas de saúde impulsionou a discussão sobre modelos que pudessem orientar o desenvolvimento de políticas, estratégias e programas, além de possibilitar a avaliação do grau de realização dos objetivos e das metas dos sistemasde saúde 10 .

Na esteira das críticas ao modelo proposto pela OMS e do debate de modelos de avaliação de desempenho implantados em diferentes países do mundo, foi iniciado no Brasil o PROADESS, atualmente denominado Programa de Avaliação do Desempenho do Sistema de Saúde, cujo marco conceitual foi publicado em 200311.

O modelo PROADESS, cuja ilustração e descrição detalhada encontra-se disponível na Internet 12,13 , considera que o desempenho do sistema de saúde brasileiro deve ser analisado em um contexto político, social e econômico que traduza a sua história, a sua conformação atual, seus objetivos e suas prioridades. Os indicadores são apresentados numa Matriz Conceitual, de dimensões e subdimensões inter-relacionadas de avaliação. A primeira dimensão está relacionada a determinantes dos problemas de saúde a serem analisados considerando o seu impacto em diferentes grupos sociais. A caracterização dos problemas de saúde conforma uma segunda dimensão da avaliação, que permite conhecer a sua magnitude e expressão em diferentes regiões geográficas e grupos sociais. Expressam as necessidades de saúde que devem orientar o desenho do sistema de saúde.

O sistema de saúde, introduzido como terceira dimensão, é avaliado em sua estrutura, ou seja, recursos humanos e materiais oferecidos, $\mathrm{e}$ 
condução, através de determinações legais, econômicas e financeiras. O desenho do sistema de saúde condiciona as possibilidades de melhor ou pior desempenho dos serviços de saúde, última dimensão de avaliação. Baseado em experiências internacionais e na literatura sobre avaliação de serviços de saúde, tendo em vista os princípios do SUS, o desempenho dos serviços pode ser avaliado levando em conta o acesso possibilitado pelo sistema; sua efetividade, dependente dos resultados alcançados pelos diversos programas; a adequação da prestação dos serviços; a eficiência e a continuidade do cuidado; os direitos das pessoas, a segurança do paciente nos diversos níveis de atenção e o nível de aceitabilidade sobre os procedimentos propostos.

A dimensão da equidade perpassa transversalmente todas as dimensões e subdimensões de avaliação.

No modelo do PROADESS, busca-se avaliar o desempenho do sistema de saúde mediante uma visão global e integradora das necessidades de saúde e das respostas produzidas pela sociedade frente a elas, concretizadas na forma do sistema de saúde. Na construção de um esquema analítico coerente com essa abordagem, um dos desafios a ser enfrentado é a identificação de espaços geográficos comparáveis - estabelecidos de forma a respeitar as especificidades, a diversidade e a complexidade das condições de saúde presentes no território nacional - com a finalidade de se constituírem nas unidades de observação e análise do desempenho dos sistemas de saúde regionalizados e do acompanhamento de sua evolução no tempo.

$\mathrm{O}$ conceito de Região de Saúde remete à constituição de uma base territorial para o planejamento de uma rede de atenção integral à saúde e esta última está claramente estabelecida no marco legal. Contudo, o Brasil tem hoje em sua malha 5.570 municípios (Instituto Brasileiro de Geografia e Estatística. Índice de organização territorial/divisão territorial. ftp://geoftp.ibge. gov.br/organizacao_territorial/divisao_territo rial, acessado em 25/Mai/2014) que se agregam em mais de 400 Regiões de Saúde 14, e a regionalização é um processo dinâmico. Um dos principais desafios metodológicos é lidar com a complexidade de avaliar o desempenho de uma rede de serviços e intervenções, homogênea na missão de prover assistência integral, mas que precisa ser desenhada para fazer frente a uma grande heterogeneidade territorial, no que se refere ao perfil de vida e saúde e, portanto, de necessidades a serem atendidas.

A estratégia usualmente aplicada para lidar com essa questão tem sido a definição de modelos de agregação em grupos mais ou menos homogêneos de municípios ou regiões, de modo a possibilitar análises comparativas. Estudiosos e autoridades sanitárias têm se valido de modelos de agregação para criar tipologias de Regiões de Saúde. Trata-se de uma empreitada das mais desafiadoras, envolvendo desde a seleção de fontes de informações confiáveis e modelos teoricamente fundamentados, até a proposição de métodos relativamente simples e robustos, de modo a favorecer a sua compreensão e utilização pelos atores ligados à gestão do SUS.

É nesse contexto que se insere o presente trabalho, que tem como objetivo apresentar uma proposta de diferenciação das atuais Regiões de Saúde no Brasil, baseada em indicadores que expressam o nível de desenvolvimento humano nos municípios que integram tais regiões. O propósito do trabalho é contribuir para a identificação de espaços geográficos comparáveis para observação, análise e acompanhamento do desempenho dos sistemas regionalizados de saúde, constituídos de forma a se aproximar pragmaticamente dos pressupostos teóricos relacionados aos determinantes sociais do processo saúdedoença presentes no PROADESS.

Essa tipologia é apresentada após uma breve contextualização do atual debate em torno da regionalização brasileira e da apresentação de três dos principais modelos utilizados para o estabelecimento de tipologias de Regiões de Saúde.

\section{Regionalização no âmbito do SUS}

Embora inscrita na Constituição Federal e nas Leis Orgânicas da Saúde, a regionalização assumiu o primeiro plano da Política Nacional de Saúde a partir da vigência da Norma Operacional de Assistência à Saúde (NOAS) 15 instituída no início deste milênio, que passou a concebê-la como estratégia necessária à descentralização e à organização da rede assistencial. Para viabilizar a regionalização, a NOAS resgatou o papel da esfera estadual de governo na construção do Plano Diretor da Regionalização, o que gerou resistências por parte dos municípios, que identificavam nesse processo uma perda de autonomia na gestão do sistema de saúde 16 .

A estratégia de regionalização se solidificou com a instituição do Pacto pela Saúde 17, sendo assumida como "eixo estruturante do Pacto de Gestão”, devendo orientar as relações entre as distintas esferas de governo. Um elemento fundamental da regionalização inscrita no pacto foi a criação dos Colegiados de Gestão Regional (CGR), concebidos "como instâncias deliberativas semelhantes às CIB, diferenciando-se pela abrangência restrita às regiões e pela obrigatorie- 
dade da participação de todos os gestores municipais envolvidos nas regiões" 6 (p. 208), onde as decisões são definidas por consenso, o que não necessariamente coincide com unanimidade.

Essa obrigatoriedade é o elemento central para o desenvolvimento de processos cooperativos, imprimindo à construção de pactos ou "pactuação" papel de destaque, em detrimento do modelo prescritivo presente na definição das normas anteriores. Porém, a experiência dos consórcios municipais de livre iniciativa mostrou que as associações voluntárias entre municípios não foram suficientes para garantir o acesso universal e, sobretudo, igualitário ao SUS, em nível regional 6 .

O Pacto pela Saúde trouxe o arcabouço institucional necessário à regionalização da saúde e, em 2011, foi publicado o Decreto no 7.508, com o ambicioso objetivo de "dar mais transparência a essa estrutura" e "garantir maior segurança jurídica na fixação das responsabilidades dos entes federativos, para que o cidadão pudesse, de fato, conhecer as ações e os serviços de saúde ofertados nas regiões de saúde e organizados em redes de atenção à saúde". Esse Decreto, que regulamenta a Lei no 8.080, trouxe algumas proposições importantes em relação à estrutura organizativa do SUS (regionalização e hierarquização), ao planejamento em saúde e à articulação interfederativa.

Em relação à estrutura, a Região de Saúde foi estabelecida como o território de referência para o planejamento e execução de ações e serviços de uma Rede de Atenção à Saúde hierarquizada, entendida como o espaço contínuo, constituído por agrupamentos de municípios limítrofes, delimitado por identidades culturais, econômicas e sociais e de redes de comunicação e infraestrutura de transportes compartilhados. Uma Rede de Atenção à Saúde é o conjunto de ações e serviços de saúde articulados em níveis de complexidade crescente, com a finalidade de garantir a integralidade da assistência à saúde. As Redes de Atenção à Saúde podem estar compreendidas no âmbito de uma Região de Saúde ou de várias delas e as regiões localizadas em áreas de fronteira com outros países deve respeitar as normas que regem as relações internacionais.

$\mathrm{O}$ acesso às ações e serviços de saúde deve ser ordenado pela atenção primária e iniciado pelas portas de entrada do SUS, completando-se na rede regionalizada e hierarquizada, de acordo com a complexidade do serviço. Da mesma forma, a integralidade da assistência à saúde "se inicia $e$ se completa na Rede de Atenção à Saúde, mediante o referenciamento do usuário na rede regional e interestadual" 5.

Outros elementos complementares à organização de rede regionalizada e hierarquizada es- tão presentes no Decreto, em especial em relação à articulação interfederativa e ao planejamento.

No que toca à articulação interfederativa, o Decreto no 7.508 reafirma as Comissões Intergestores como fóruns de pactuação da organização e funcionamento das ações e serviços integrados em redes. Além das comissões nacional e estaduais (CIT e CIB), cria as Comissões Intergestores Regionais (CIR), que substituem os CGR. Nesta esfera, a introdução de uma nova forma de relação entre os gestores dos distintos níveis de governo, o Contrato Organizativo de Ação Pública da Saúde (COAP), altera o caráter da pactuação preconizado pelo Pacto pela Saúde e passa a estabelecer mecanismos de contratualização entre os gestores do SUS. Por meio do COAP, todos os municípios devem assinar a sua adesão às novas Regiões de Saúde e estão obrigados a prestar contas da sua atuação. O desempenho deverá ser aferido com base em "indicadores nacionais de garantia de acesso", que servirão como "parâmetro para a avaliação do desempenho da prestação das ações e dos serviços definidos no COAP em todas as Regiões de Saúde, considerando-se as especificidades municipais, regionais e estaduais" 5.

Finalmente, o Mapa da Saúde foi estabelecido como principal recurso de identificação das necessidades de saúde, orientando o planejamento integrado dos entes federativos e contribuindo para o estabelecimento de metas compartilhadas. É definido como a descrição geográfica da distribuição de recursos humanos e de ações e serviços ofertados pelo SUS e pela iniciativa privada, considerando-se a capacidade instalada existente, os investimentos e o desempenho aferido pelos indicadores de saúde do sistema. Indica, também, que o planejamento é obrigatório para os entes públicos e deve induzir políticas para o setor privado.

No final de 2013, dois anos após a promulgação do Decreto, o COAP havia sido oficializado em apenas dois estados (Ceará e Mato Grosso do Sul), o que pode ser um indício do modo como os atores locais encararam a possibilidade de contratualização das responsabilidades dos sistemas de saúde neste nível. Apesar dos avanços no processo de regionalização, ainda persistem vários desafios equitativos: as enormes desigualdades entre as necessidades em saúde e a distribuição da rede de serviços podem estar refletidas na presença de regiões destituídas da rede necessária para uma atenção integral à saúde (vazios assistenciais).

Para subsidiar o processo de regionalização e conferir maior transparência à estrutura de saúde e segurança jurídica na fixação das responsabilidades pela oferta de serviços, como determina o Decreto no 7.508, é fundamental a construção 
de modelos de monitoramento e avaliação do desempenho do sistema de saúde regionalizado, passíveis de serem incorporados pelos atores locais, como instrumentos para o planejamento. Parte desse modelo inclui a definição de unidades territoriais homogêneas para a observação e análise. Três modelos de agregação de regiões de saúde com maior visibilidade nos últimos anos serão descritos a seguir: o oficial, proposto pelo Ministério da Saúde como parte do referencial do Índice de Desempenho do SUS (IDSUS) 18,19, o desenvolvido por um grupo multi-institucional, coordenado por Ana Luiza d'Ávila Viana e Luciana Dias de Lima para a análise da evolução do processo de regionalização no Brasil 20,21 e um modelo utilizado pela Fundação Seade vinculada à Secretaria Estadual de Planejamento e Desenvolvimento Regional do Estado de São Paulo 22.

\section{Tipologias para avaliar o desempenho do SUS nas Regiões de Saúde}

O IDSUS foi lançado em 2011, como parte do sistema de avaliação para qualificação do SUS 19 e a sua construção pode ser sintetizada em quatro estágios, o primeiro de seleção, construção e valoração dos indicadores simples para avaliação do desempenho, e os demais de aplicação de metodologia de construção de indicadores compostos ou índices, em camadas de agregação consecutivas até culminar no IDSUS 18.

Considerando que seria inadequado fazer uma classificação, utilizando apenas o valor do IDSUS de todos os municípios brasileiros, o modelo avaliativo desenvolvido pelo Ministério da Saúde conta também com uma etapa de caracterização dos municípios brasileiros e de seu agrupamento, em seis Grupos Homogêneos de Municípios. A estratégia metodológica desenvolvida pelo Ministério da Saúde preconiza que uma análise comparativa do desempenho do SUS entre os municípios seria recomendável apenas no interior de cada um desses grupos 18 .

Embora a descrição mais detalhada do método de cálculo da nota do IDSUS pudesse ser ilustrativa de sua complexidade, ela foge ao escopo deste trabalho. É a etapa de criação de tipologias de grupos homogêneos de municípios, a que mais diretamente diz respeito ao seu objetivo.

Para encontrar os Grupos Homogêneos de Municípios, foram utilizados três índices, construídos pela ponderação de indicadores simples por meio do método Análise de Componentes Principais (ACP): o Índice de Desenvolvimento Econômico (IDSE), o Índice de Condições de Saúde (ICS) e o Índice de Estrutura do Sistema de Saúde do Município (IESSM).
Primeiramente, os municípios foram separados em dois subconjuntos, com base no resultado do IESSM e na capacidade de internação hospitalar. Em seguida os dois subconjuntos foram submetidos à análise de agrupamento (cluster analysis) pelo método K-Médias, resultando em seis grupos homogêneos, de acordo com a classificação recebida nos índices IDSE, ICS e IESSM.

Desde a sua divulgação, o IDSUS vem sendo objeto de debates por parte de estudiosos $23,24,25$. Mesmo que seja reconhecido como fruto de um esforço necessário para a definição de mecanismos de monitoramento e avaliação, vem sofrendo críticas que dizem respeito, entre outras coisas, à seleção dos indicadores que o compõem, à sua aplicação em diferentes contextos brasileiros e ao questionamento do uso de indicadores sintéticos para avaliar o desempenho do SUS. A descrição do método deixou óbvia também a complexidade do cálculo, resultando em pouca transparência, especialmente para gestores.

Com o objetivo de elucidar os condicionantes estruturais do processo recente de regionalização brasileiro, outros autores 20,21 apresentaram uma tipologia nacional das regiões geográficas configuradas pelos CGR, grupamentos de municípios que se configuram como instâncias de cogestão dos espaços regionais, formalmente constituídos até janeiro de 2010; antes, portanto, da publicação do Decreto no 7.508 .

Em uma das etapas do trabalho, os CGR foram classificados de forma independente em cinco grupos, de acordo com o grau de desenvolvimento e perfil do sistema de saúde. Para tanto, foram selecionadas 37 variáveis, inicialmente classificadas em três dimensões: socioeconômica, condições de saúde da população e complexidade do sistema de saúde. Por meio de procedimentos de análise fatorial, esse extenso número de variáveis foi reduzido para 10 fatores, entre os quais 3 se mostraram mais discriminantes e foram utilizados para os procedimentos de análise de agrupamento.

A tipologia final resultou em cinco grupos de regiões, caracterizados pela combinação de diferentes níveis de desenvolvimento econômico, desenvolvimento humano e de complexidade de serviços de saúde. Ressalte-se que o fator denominado como desenvolvimento humano está relacionado aos componentes do Índice de Desenvolvimento Humano (IDH) do Programa das Nações Unidas para o Desenvolvimento (PNUD), isto é, aos níveis de renda, escolaridade e longevidade.

Recentemente, essa tipologia foi atualizada, passando a considerar as Regiões de Saúde no lugar das CGR. Mantendo os mesmos parâmetros norteadores, o número de componentes foi 
diminuído para uma "operacionalização mais amigável”, segundo eles, adequada a possíveis mudanças no desenho das Regiões de Saúde, passíveis de ocorrer ao longo do tempo. Foram, adicionalmente, incluídas informações referentes ao Censo Demográfico de 2010, não disponíveis no momento do estudo original, em substituição às informações provenientes do IDH 26.

O Índice Paulista de Responsabilidade Social (IPRS), proposto durante o Fórum São Paulo Século XXI, realizado em 2000, lançou, em 2012, a sua sétima edição ${ }^{22}$. Calculado bianualmente para os 645 municípios do estado, é um indicador inspirado no IDH do PNUD. Preserva no seu cálculo as 3 dimensões consagradas pelo IDH (renda, longevidade e escolaridade), mas incorpora duas alterações importantes: utiliza fontes de dados de maior periodicidade e cobertura municipal, possibilitando análises mais ágeis, e utiliza variáveis distintas daquelas empregadas no cálculo do IDH, especialmente com a finalidade de aumentar o seu poder discriminante para o estado mais rico da nação.

Para cada uma dessas dimensões foi selecionado um conjunto de variáveis e, por um modelo de análise fatorial, foi criado um indicador sintético de cada dimensão. A abordagem metodológica do IDH não foi usada na construção dos grupos de municípios do IPRS, já que este último parte do pressuposto de que a mensuração da qualidade de vida deve considerar vários aspectos não transferíveis entre si, isto é, que nenhuma das dimensões que constituem os componentes do IDH pode substituir outra. Assim, pela aplicação de uma análise de agrupamentos, o IPRS agregou os municípios paulistas em 5 grupos, caracterizados por combinações dos indicadores sintéticos de riqueza, longevidade e escolaridade. Vale destacar que, diversamente do método proposto no estudo multi-institucional 20,21, a proposta do IPRS não considera a estrutura de saúde na caracterização dos agrupamentos.

\section{Uma tipologia das Regiões de Saúde no Brasil, baseada no desenvolvimento humano}

Conforme mencionado, a tipologia proposta nesta seção aproxima-se dos pressupostos teóricos relacionados aos determinantes sociais do processo saúde-doença adotados no PROADESS. É compatível, também, com categorias de análise propostas pela corrente teórico-metodológica dos determinantes sociais da saúde tais como características populacionais, iniquidades sociais, condições de vida, necessidades e contextos dos problemas de saúde 27,28.

Na construção da tipologia das Regiões de Saúde, os componentes do Índice de Desenvolvimento Humano Municipal (IDHM) foram utilizados como proxy de determinantes sociais da saúde e o modelo de análise de agrupamento denominado K-Médias foi utilizado para a definição de grupos de regiões homogêneas.

O IDHM é uma adaptação do IDH Global, desenvolvido pelo PNUD 29, cuja divulgação foi operacionalizada pela plataforma Atlas do Desenvolvimento Humano no Brasil 2013, sendo de responsabilidade do PNUD Brasil, do Instituto de Pesquisa Econômica Aplicada e da Fundação João Pinheiro. A sua construção para o ano de 2013 foi realizada por meio de um extenso trabalho de dois anos, envolvendo três instituições e mais de 40 especialistas brasileiros.

O IDHM segue as mesmas três dimensões do IDH Global - longevidade, educação e renda, mas adequa a metodologia global ao contexto brasileiro e à disponibilidade, qualidade e periodicidade de indicadores nacionais. O IDHM Longevidade (IDHM-L) considera a esperança de vida ao nascer, o mesmo indicador do IDH Global. Já o IDHM Educação (IDHM-E) considera a escolaridade da população adulta e o fluxo escolar da população jovem. Assim como o IDH Global, é composto por um indicador sobre a população adulta e um referente à população em idade escolar, entretanto, as variáveis são diferentes. O IDHM Renda (IDHM-R) é definido pelo cálculo da renda municipal per capita, e o IDH Global utiliza, a partir de 2010, a renda nacional bruta per capita.

A exemplo do IPRS, considerou-se neste trabalho que a mensuração da qualidade de vida deve ter em conta vários aspectos não transferíveis entre si, ou seja, que nenhuma das dimensões do IDHM pode substituir a outra e, por esse motivo, foram utilizados os índices desagregados do IDHM, vale dizer, o IDHM-L, o IDHM-E e o IDHM -R, cujos dados foram obtidos na plataforma Atlas Brasil 2013 (http://www.atlasbrasil.org.br/2013/).

Pela base de dados, os indicadores de cada dimensão do IDHM foram calculados para cada Região de Saúde, por meio de agregações dos indicadores municipais ponderados pelas respectivas razões entre os tamanhos populacionais dos municípios e o total populacional da Região de Saúde nas faixas etárias específicas. A partir dessa etapa, os valores desses indicadores foram utilizados nas fórmulas originais de cálculo do IDHM 30 propostas pelo PNUD, que geraram os resultados de cada uma das suas dimensões. A Tabela 1 descreve um exemplo do processo de cálculo. 
Exemplo do processo de obtenção dos valores das dimensões do Índice de Desenvolvimento Humano Municipal (IDHM): educação (IDHM-E), longevidade (IDHM-L) e renda (IDHM-R), para a Região de Saúde R (hipotética).

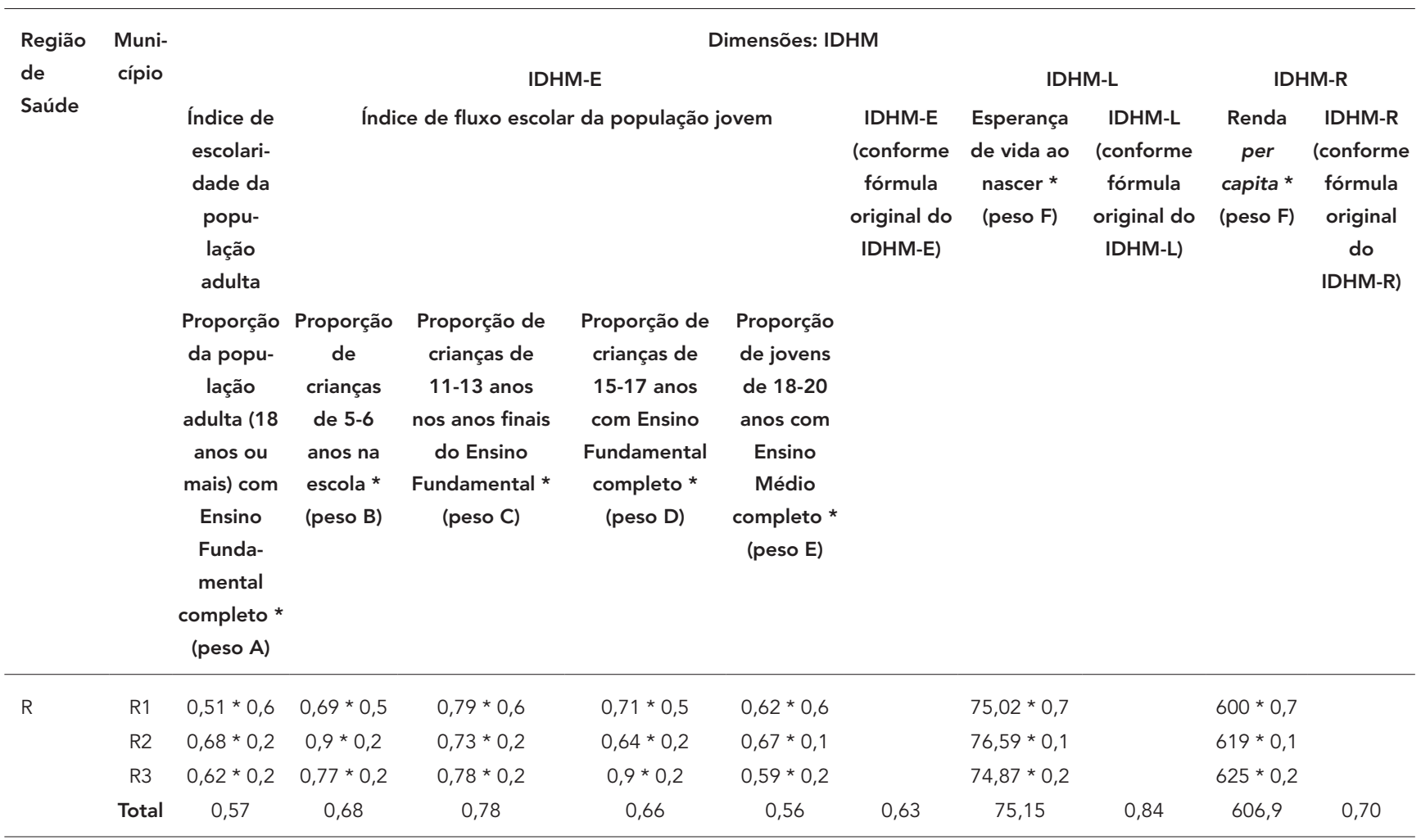

Peso A: peso em relação ao tamanho da população com 18 anos ou mais; Peso B: peso em relação ao tamanho da população de 5-6 anos; Peso C: peso em relação ao tamanho da população de 11-13 anos; Peso D: peso em relação ao tamanho da população de 15-17 anos; Peso E: peso em relação ao tamanho da população de 18-20 anos; Peso F: peso em relação ao tamanho da população.

Fonte: elaboração própria.

O desenho das Regiões de Saúde, isto é, a lista de Regiões, segundo os municípios integrantes, obedece à configuração utilizada na definição de objetivos e metas do Ministério da Saúde, no período 2013-2015, informada ao PROADESS 14.

O agrupamento de Regiões de Saúde segundo combinações de longevidade, riqueza e escolaridade foi determinado pelo método K-Médias, um método não hierárquico e iterativo que, para a formação de conglomerados, realiza uma partição dos elementos em k grupos, onde k é um número que deve ser fixado a priori. Neste trabalho o número de grupos foi fixado em 5, o mesmo número de tipos utilizados no IDHM. A análise de agrupamentos identifica perfis semelhantes em um conjunto de observações, no caso Regiões de Saúde, segundo um conjunto de variáveis selecionadas, no caso IDHM-E, IDHM-L e IDHM-R. Dessa forma, pela identificação dos padrões de semelhanças e diferenças, cada grupo de regiões constituído é internamente o mais homogêneo possível, ao mesmo tempo em que os grupos são diferentes entre si, em relação às combinações de escolaridade, longevidade e renda.

\section{Resultados e discussão}

Os 5 grupos de Regiões de Saúde resultantes da análise de agrupamento por dimensões do desenvolvimento humano estão descritos na Tabela 2. As Regiões de Saúde foram classificadas nos tipos 1 a 5, com padrão decrescente, isto é, o Grupo 1 representa as regiões com os melhores indicadores de desenvolvimento humano e o grupo 5 reúne aquelas com os piores.

Cerca de metade das Regiões de Saúde (53\%) foram classificadas com os melhores índices das dimensões de desenvolvimento humano, respectivamente $25 \%$ no grupo de tipo 1 e $28 \%$ no grupo de tipo 2 e, no extremo oposto, cerca de $32 \%$ das Regiões de Saúde foram classificadas nos grupos com os piores índices: $17 \%$ no tipo 4 e $15 \%$ no tipo 5. Quinze por cento das Regiões de Saúde se 
Tabela 2

Grupos de Regiões de Saúde por dimensões do desenvolvimento humano, 2013

\begin{tabular}{|c|c|c|c|c|c|c|}
\hline \multicolumn{2}{|c|}{ Dimensões do Desenvolvimento Humano } & \multicolumn{5}{|c|}{ Agrupamento de Regiões de Saúde } \\
\hline & & \multirow{2}{*}{$\begin{array}{c}5 \\
(15 \%)\end{array}$} & \multirow{2}{*}{$\begin{array}{c}4 \\
(17 \%) \\
58 \%\end{array}$} & \multirow{2}{*}{$\begin{array}{c}3 \\
(15 \%)\end{array}$} & \multirow{2}{*}{$\begin{array}{c}\begin{array}{c}2 \\
(28 \%)\end{array} \\
0 \%\end{array}$} & \multirow{2}{*}{$\begin{array}{c}1 \\
(25 \%) \\
0 \%\end{array}$} \\
\hline IDHM-R & Muito baixo & & & & & \\
\hline & Baixo & $6 \%$ & $42 \%$ & $94 \%$ & $12 \%$ & $0 \%$ \\
\hline & Médio & $0 \%$ & $0 \%$ & $5 \%$ & $74 \%$ & $14 \%$ \\
\hline & Alto & $0 \%$ & $0 \%$ & $0 \%$ & $14 \%$ & $86 \%$ \\
\hline & Muito alto & $0 \%$ & $0 \%$ & $0 \%$ & $0 \%$ & $0 \%$ \\
\hline \multirow[t]{5}{*}{ IDHM-E } & Muito baixo & $97 \%$ & $51 \%$ & $9 \%$ & $0 \%$ & $0 \%$ \\
\hline & Baixo & $3 \%$ & $49 \%$ & $77 \%$ & $18 \%$ & $0 \%$ \\
\hline & Médio & $0 \%$ & $0 \%$ & $14 \%$ & $74 \%$ & $6 \%$ \\
\hline & Alto & $0 \%$ & $0 \%$ & $0 \%$ & $8 \%$ & $94 \%$ \\
\hline & Muito alto & $0 \%$ & $0 \%$ & $0 \%$ & $0 \%$ & $0 \%$ \\
\hline \multirow[t]{5}{*}{ IDHM-L } & Muito baixo & $96 \%$ & $59 \%$ & $2 \%$ & $0 \%$ & $0 \%$ \\
\hline & Baixo & $4 \%$ & $41 \%$ & $91 \%$ & $13 \%$ & $1 \%$ \\
\hline & Médio & $0 \%$ & $0 \%$ & $8 \%$ & $58 \%$ & $32 \%$ \\
\hline & Alto & $0 \%$ & $0 \%$ & $0 \%$ & $30 \%$ & $68 \%$ \\
\hline & Muito alto & $0 \%$ & $0 \%$ & $0 \%$ & $0 \%$ & $0 \%$ \\
\hline
\end{tabular}

IDHM: Índice de Desenvolvimento Humano Municipal: educação (IDHM-E), longevidade (IDHM-L) e renda (IDHM-R).

Fonte: elaboração própria, com base no Atlas do Desenvolvimento Humano no Brasil 201330.

localizaram no grupo do tipo 3. Especificamente nos grupos dos tipos de 3 a 5 , a maior parte das Regiões de Saúde apresentou índices baixos ou muito baixos. Já nos grupos do tipo 1 e 2, a maior parte apresentou índices médios ou altos. Em última análise, percebe-se que cerca de metade das Regiões de Saúde brasileiras foram classificadas em grupos com índices de longevidade, renda e escolaridade baixos ou muito baixos e a outra metade em grupos com índices médios ou altos.

A Tabela 3 evidencia que as regiões Sul, Centro-oeste e Sudeste apresentaram a maior concentração de Regiões de Saúde entre os tipos 1 e 2 , respectivamente $96,5 \%, 85,3 \%$ e $83,4 \%$. O inverso é observado na Região Nordeste, que apresentou 78,9\% de suas Regiões de Saúde classificadas entre os tipos 4 e 5 . A Região Norte, apesar de apresentar $46 \%$ de suas Regiões de Saúde também entre os tipos 4 e 5, destaca-se da Região Nordeste por apresentar $15,3 \%$ de suas regiões no tipo 3.

A Figura 1 completa a análise, oferecendo uma perspectiva espacial dos resultados. Observa-se que, de modo geral, os centros urbanos/ capitais pertencem ao grupo dos mais desenvolvidos (1 e 2). Nas grandes regiões Sudeste, Sul e Centro-oeste, a maioria das Regiões de Saúde pertence aos dois melhores tipos de classificação e poucas pertencem a uma categoria inter- mediária ou baixa ( 3 e 4), e a maioria delas se localiza perto das fronteiras com regiões Norte e Nordeste. Não há Regiões de Saúde de tipo 5 no Sul e Centro-oeste e, no Sudeste há apenas uma. Conforme mencionado, nas regiões Nordeste e Norte, com exceção de algumas capitais, a maioria das unidades pertence às últimas categorias (4 e 5) e a Região Norte apresenta uma maior concentração de Regiões de Saúde do tipo 3. Percebe-se que as regiões do tipo 5 encontram-se no semiárido nordestino e na floresta amazônica.

O desenho das regiões de saúde segundo os tipos encontrados nos resultados deste trabalho não é comparável aos modelos de agregação disponibilizados até o momento na literatura ou por órgãos oficiais brasileiros, apresentados anteriormente. O modelo do IDSUS considera a distribuição de municípios (e não de Regiões de Saúde), trabalha com 6 tipos ou agrupamentos e incorpora a estrutura de serviços de saúde entre as variáveis que definem a tipologia; o modelo do estudo multi-institucional considerou os CGR, nomenclatura definida no Pacto pela Saúde, vigente até a promulgação do Decreto no 7.508 de 2011, mas não incluiu em seu modelo os estados do Acre, Amazonas, Roraima, Maranhão e parte do Piauí. Ainda que a atualização recentemente incorporada considere a distribuição das Regiões de Saúde, o modelo, assim 
Tabela 3

Número e percentual de Regiões de Saúde por Grandes Regiões do Brasil, segundo os grupos de desenvolvimento humano, 2013.

\begin{tabular}{|c|c|c|c|c|c|c|c|c|c|c|c|c|}
\hline & \multicolumn{2}{|c|}{1} & \multicolumn{2}{|c|}{2} & \multicolumn{2}{|c|}{3} & \multicolumn{2}{|c|}{4} & \multicolumn{2}{|c|}{5} & \multicolumn{2}{|c|}{ Total } \\
\hline & $n$ & $\%$ & $n$ & $\%$ & $n$ & $\%$ & $n$ & $\%$ & $n$ & $\%$ & $n$ & $\%$ \\
\hline Norte & 1 & 1,6 & 12 & 19,7 & 20 & 32,8 & 14 & 23,0 & 14 & 23,0 & 61 & 100,0 \\
\hline Nordeste & 4 & 2,9 & 4 & 2,9 & 21 & 15,3 & 56 & 40,9 & 52 & 38,0 & 137 & 100,0 \\
\hline Sudeste & 76 & 48,4 & 55 & 35,0 & 17 & 10,8 & 8 & 5,1 & 1 & 0,6 & 157 & 100,0 \\
\hline Sul & 26 & 45,6 & 29 & 50,9 & 2 & 3,5 & 0 & 0,0 & 0 & 0,0 & 57 & 100,0 \\
\hline Centro-oeste & 4 & 11,8 & 25 & 73,5 & 5 & 14,7 & 0 & 0,0 & 0 & 0,0 & 34 & 100,0 \\
\hline Total & 111 & 24,9 & 125 & 28,0 & 65 & 14,6 & 78 & 17,5 & 67 & 15,0 & 446 & 100,0 \\
\hline
\end{tabular}

Fonte: elaboração própria, com base no Atlas do Desenvolvimento Humano no Brasil 201330.

\section{Figura 1}

Classificação das Regiões de Saúde por Grandes Regiões do Brasil, segundo os grupos de desenvolvimento humano, 2013.

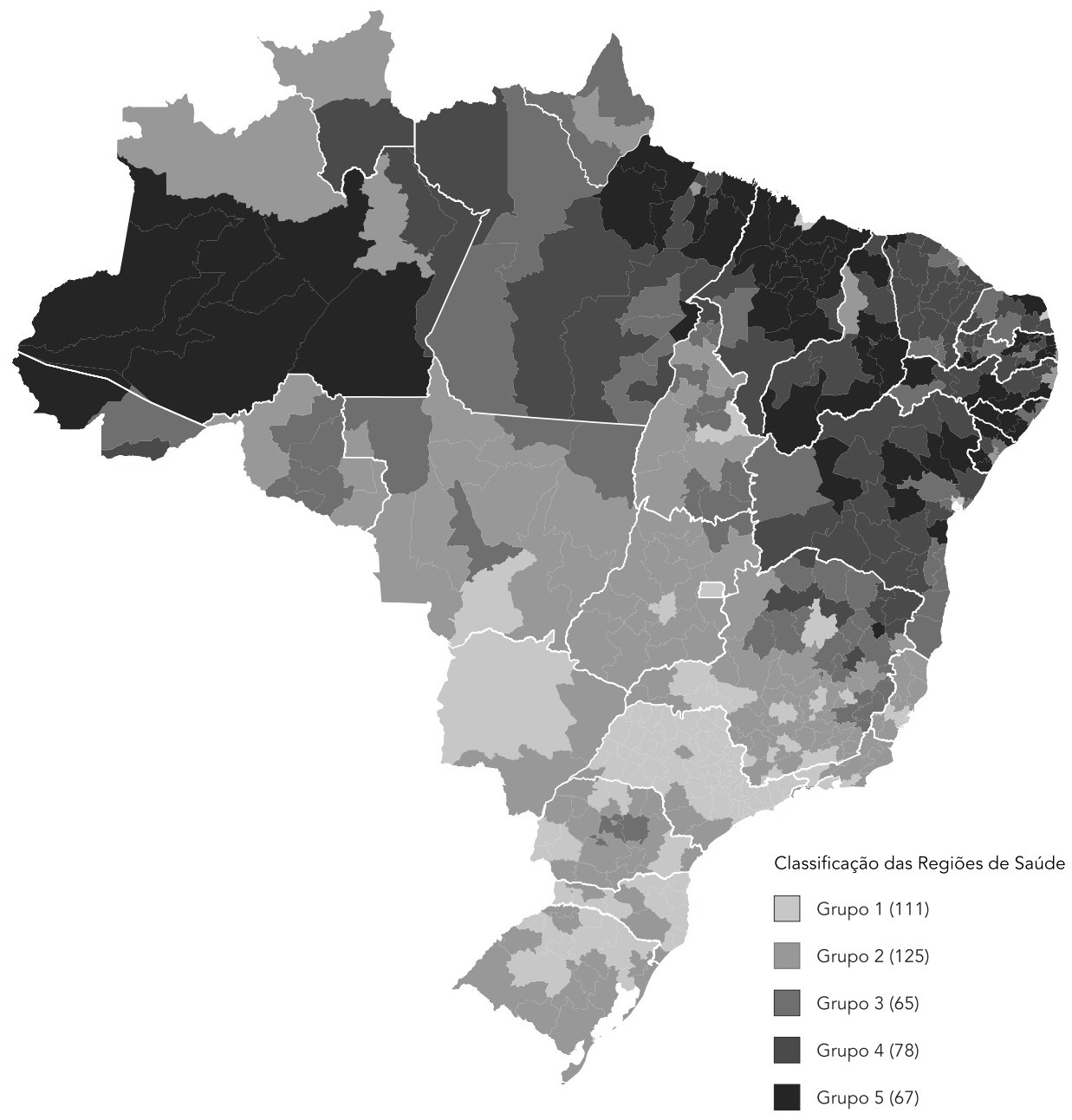

Fonte: elaboração própria, com base no Atlas do Desenvolvimento Humano no Brasil 201330. 
como o IDSUS, incorpora a estrutura de serviços de saúde às variáveis definidoras da tipologia. Finalmente, o modelo utilizado no IPRS se refere a municípios do Estado de São Paulo.

Por outro lado, os procedimentos analíticos para definir agrupamentos são semelhantes em todos os trabalhos. As principais diferenças verificadas são em relação aos indicadores selecionados para compor o modelo. Nesse sentido, os procedimentos apresentados nesta proposta assemelham-se àqueles utilizados no IPRS, que também trabalha com os componentes do IDHM, embora no caso deste trabalho os valores dos componentes do IDH tenham sido extraídos diretamente da Plataforma Brasil, sem as adaptações nos indicadores realizadas pela Fundação Seade. No entanto, tais adaptações, suportadas pela qualidade do sistema de informações do Estado e realizadas para aumentar o poder discriminante dos indicadores a fim de diferenciar municípios com bons níveis socioeconômicos, não necessariamente se aplicam aos demais estados da nação. As demais tipologias são mais complexas, utilizam um maior número de indicadores, ambas incluem indicadores relacionados aos serviços de saúde e a utilizada no IDSUS é a mais intricada.

\section{Conclusões}

A tipologia apresentada oferece um modelo de agrupamento de Regiões de Saúde homogêneas, coerente com os pressupostos teóricos do PROADESS, para servirem de unidades de observação e análise do desempenho do SUS. A análise de desempenho considerando os diferentes tipos estabelecidos pode, por sua vez, impactar positivamente a capacidade de o sistema de saúde brasileiro diminuir desigualdades, já que, levando em conta o conceito de equidade viabiliza o direcionamento de investimentos para as éreas incluídas nos piores grupamentos ou tipos, subsidiando o processo de regionalização.

A opção por indicadores e métodos de agregação bem estabelecidos, utilizando bases de dados acessíveis, disponíveis para todo o Brasil, atendendo da melhor forma possível aos critérios de simplicidade, transparência, qualidade e poder discriminatório dos indicadores, tende a favorecer a sua compreensão e utilização pelos atores ligados à gestão do SUS.

Tendo em vista o dinamismo da regionalização e o desafio representado pela seleção de variáveis e modelos de definição de tipologias, são desdobramentos desejáveis, de um lado, uma análise espacial comparativa com tipologias resultantes das diferentes metodologias passíveis de aplicação às Regiões de Saúde brasileiras e, de outro, avaliações sobre o desempenho de sistemas de saúde nos grupos com características socioeconômicas homogêneas. 


\section{Resumen}

Este trabajo presenta una propuesta para la diferenciación de las Regiones de Salud en Brasil, basado en el desarrollo humano, lo que contribuye a la identificación de áreas geográficas comparables para la observación, el análisis y el seguimiento del desempeño de los sistemas sanitarios regionalizados. Se calcularon los valores de las dimensiones del Índice de Desarrollo Humano Municipal para las Regiones de Salud, a partir de los datos municipales, ponderados por su tamaño poblacional. La clasificación de las Regiones de Salud en 5 grupos, de acuerdo a las combinaciones de longevidad, riqueza y educación, se determinó por el método $K$-medias. La mitad de las Regiones de Salud de Brasil fueron clasificadas como tipo 1 y grupo 2, y la otra mitad en grupos de dos tipos 3-5. La tipología presentada proporciona un modelo de agrupación de regiones sanitarias homogéneas, en consonancia con los supuestos teóricos de PROADESS. La elección de los indicadores y los métodos de agregación bien establecidos tienden a facilitar su comprensión y su utilización por los gestores del Sistema Único de Salud brasileño (SUS).

Regionalización; Evaluación en Salud;

Desarrollo Humano

\section{Colaboradores}

C. M. R. Duarte participou da concepção do projeto, análise e interpretação dos resultados e foi responsável pela redação do artigo, revisão crítica do conteúdo intelectual e aprovação final da versão publicada. M. M. Pedroso participou da concepção do projeto, análise e interpretação dos resultados e contribuiu na redação do artigo, revisão crítica do conteúdo intelectual e aprovação final da versão publicada. J. G. Bellido e R. S. Moreira participaram da concepção do projeto, análise e interpretação dos resultados e contribuíram na redação do artigo e aprovação final da versão publicada. F. Viacava participou da concepção do projeto, revisão crítica do conteúdo intelectual e aprovação final da versão publicada.

\section{Agradecimentos}

Agradecemos aos pareceristas que colaboraram para o aprimoramento deste artigo.

\section{Referências}

1. Brasil. Constituição da República Federativa do Brasil. Brasília: Senado Federal; 1988.

2. Brasil. Lei no 8.080, de 19 de setembro de 1990 . Dispõe sobre as condições para a promoção, proteção e recuperação da Saúde, a organização e financiamento dos serviços correspondentes e dá outras providências. Diário Oficial da União 1990; 19 set.

3. Brasil. Lei no 8.142, de 28 de dezembro de 1990. Dispõe sobre a participação da comunidade na gestão do Sistema Único de Saúde - SUS e sobre as transferências intragovernamentais de recursos financeiros na área de saúde e dá outras providências. Diário Oficial da União 1990; 28 dez.

4. Mendes EV. As redes de atenção à saúde. Brasília: Organização Pan-Americana da Saúde; 2011.

5. Brasil. Decreto Federal no 7.508 , de 28 de junho de 2011. Regulamenta a Lei no 8.080, de 19 de setembro de 1990, para dispor sobre a organização do Sistema Único de Saúde - SUS, o planejamento da saúde, a assistência à saúde e a articulação interfederativa, e dá outras providências. Diário Oficial da União 2011; 29 jun.

6. Dourado DA, Elias PEM. Regionalização e dinâmica política do federalismo sanitário brasileiro. Rev Saúde Pública 2011; 45:204-11.
7. Levi ML, Scatena JHG. Evolução recente do financiamento do SUS e considerações sobre o processo de regionalização. In: Viana ALA, Lima LD, organizadores. Regionalização e relações federativas na política de saúde do Brasil. Rio de Janeiro: Contra Capa; 2011. p. 81-113.

8. Hartz ZMA, Silva LMV, organizadoras. Avaliação em saúde: dos modelos teóricos à prática na avaliação de programas e sistemas de saúde. Salvador: EDUFBA/Rio de Janeiro: Editora Fiocruz; 2005.

9. World Health Organization. World Health Report 2000. Health systems: improving performance. Geneva: World Health Organization; 2000.

10. Organización Panamericana de la Salud. Evaluación y mejora del desempeño de los sistemas de salud en la región de las Américas. Washington DC: Organización Panamericana de la Salud; 2001.

11. Almeida C. Projeto: desenvolvimento de metodologia de avaliação do desempenho do sistema de saúde brasileiro (PRO-ADESS). Relatório final. http:// www.proadess.icict.fiocruz.br/relatoriofinal.pdf (acessado em 21/Nov/2014).

12. Viacava F, coordenador. PROADESS - Avaliação de Desempenho do Sistema de Saúde Brasileiro: indicadores para monitoramento. Relatório. Rio de Janeiro; 2012. http://www.proadess.icict.fiocruz.br/ index.php?pag=produ (acessado em 21/Nov/2014) 
13. PROADESS. Matriz de dimensões do desempenho do sistema de saúde. http://www.proadess.icict. fiocruz.br/index.php?pag=matp (acessado em 21/ Nov/2014).

14. Instituto de Comunicação e Informação Científica e Tecnológica em Saúde, Fundação Oswaldo Cruz. Regiões de Saúde. PROADESS - Avaliação de Desempenho do Sistema de Saúde Brasileiro. http:// www.proadess.icict.fiocruz.br/index.php?pag=ma trs (acessado em 25/Mai/2014).

15. Departamento de Descentralização da Gestão da Assistência, Secretaria de Assistência à Saúde, Ministério da Saúde. Regionalização da assistência à saúde: aprofundando a descentralização com equidade no acesso. Norma Operacional da Assistência à Saúde: NOAS-SUS 01/02 (Portaria MS/GM no 373, de 27 de fevereiro de 2002 e regulamentação complementar). 2a Ed. Brasília: Ministério da Saúde; 2002.

16. Arretche M. Políticas sociais no Brasil: descentralização em um estado federativo. Rev Bras Ciênc Soc 1999; 14:111-41.

17. Ministério da Saúde. Portaria no 399/GM, de 22 de fevereiro de 2006. Divulga o Pacto pela Saúde 2006 - Consolidação do SUS e aprova as Diretrizes Operacionais do referido Pacto. Diário Oficial da União 2006; 22 fev.

18. Reis AT, Amaral MA, Oliveira PTR. IDSUS: Índice de Desempenho do Sistema Único de Saúde. http://portal.saude.gov.br/portal/arquivos/pdf/ IDSUS_29-09-12.pdf (acessado em 30/Mai/2014).

19. Reis AT, Oliveira PTR, Sellera PE. Sistema de avaliação para a qualificação do Sistema Único de Saúde (SUS). RECIIS 2012; 6(2). http://www.reciis.icict. fiocruz.br/index.php/reciis/article/view/543.

20. Viana, ALA, Lima LD, Ferreira MP. Condicionantes estruturais da regionalização na saúde: tipologia dos Colegiados de Gestão Regional. Ciênc Saúde Coletiva 2010; 15:2317-26.

21. Ferreira MP, Dini NP. Tipologia nacional dos colegiados de gestão regional. In: Viana ALA, Lima LD, organizadores. Regionalização e relações federativas na política de saúde do Brasil. Rio de Janeiro: Contra Capa; 2011. p. 67-80.
22. Ferreira MP, coordenador. O estado dos municípios 2008-2010. Índice Paulista de Responsabilidade Social. Metodologia. http://www.iprsipvs. seade.gov.br/view/pdf/iprs/IPRS_2012_v2_ RMSP.pdf.

23. Viacava F, Porto SM, Laguardia J, Ugá MAD. Avaliação do desempenho do SUS. http://cebes.org br/2012/03/avaliacao-do-desempenho-do-sus/ (acessado em 21/Set/2013).

24. Noronha JC. Impacto do IDSUS. http://www.canal. fiocruz.br/video/index.php?v=impactos-do-idsus (acessado em 25/Mai/2014).

25. Mendes AN, Tanaka OY. Considerações sobre o IDSUS. http://www.fsp.usp.br/site/noticias/mos trar/1986 (acessado em 30/Mai/2014).

26. Pesquisa Região e Redes. O caminho para a universalização da saúde no Brasil. Metodologia 2013. http://www.resbr.net.br/indicadores/view/pdf/ relatorio_tipologias_versaojul2014.pdf (acessado em 28/Nov/2014).

27. Castellanos PL. Análisis de situación de salud de poblaciones. In: Martínez-Navarro F, organizador. Vigilancia epidemiológica. Madrid: McGraw-Hill/ Interamericana; 2004; p. 193-213.

28. Rojas LI. La diferenciación territorial de la salud en la recuperación de los contextos. In: Barcellos C, organizador. A geografia e o contexto dos problemas de saúde. Rio de Janeiro: ABRASCO; 2008. p. 87-106.

29. Programa das Nações Unidas para o Desenvolvimento. Atlas do Desenvolvimento Humano no Brasil 2013. http://www.pnud.org.br/ IDH/Atlas2013.aspx?indiceAccordion $=1 \&$ li $=\mathrm{li}_{-}$ Atlas2013 (acessado em 21/Mar/2014).

30. Programa das Nações Unidas para o Desenvolvimento. Atlas do Desenvolvimento Humano Brasil. O Atlas. Metodologia. http://www.atlasbrasil.org. br/2013/pt/o_atlas/metodologia/construcao-das unidades-de-desenvolvimento-humano/ (acessado em 21/Mar/2014).

Recebido em 22/Jul/2014

Versão final reapresentada em 18/Dez/2014 Aprovado em 02/Mar/2015 